\title{
Revisión de revistas científicas de dietética y nutrición
}

\section{Review of Scientific Journals on Dietetics and Nutrition}

Según la última edición (2011) de la herramienta Journal Citation Reports (www.goo.gl/AjVPP), publicación anual del Institute of Scientific Information, las cuatro revistas científicas de mayor impacto en el ámbito de la nutrición y dietética ("Nutrition \& Dietetics") son, en orden:

\section{Progress in Lipid Research \\ Annual Review of Nutrition \\ The American Journal of Clinical Nutrition \\ Nutrition Research Reviews}

A continuación se ofrece una breve selección que el Comité Editorial ha realizado de los artículos aparecidos en dichas revistas durante los meses de agosto y septiembre de 2012 (ambos incluidos). De algunos de ellos se ofrece un breve resumen.

También se incluye en esta selección, pese a que su impacto es menor (ocupa el puesto 16), Journal of the Academy of Nutrition and Dietetics (antigua Journal of the American Dietetic Association), ya que los socios de la AED-N tienen acceso gratuito a sus contenidos (se puede solicitar escribiendo a jada@grep-aedn.es). Se suele poder consultar gratuitamente los textos completos de las demás revistas desde ordenadores de facultades que imparten enseñanzas relacionadas con la nutrición humana y dietética.

\section{Revista Progress in Lipid Research}

- Otocka-Kmiecik A, Mikhailidis DP, Nicholls SJ, Davidson M, Rysz J, Banach M. Dysfunctional HDL: A novel important diagnostic and therapeutic target in cardiovascular disease? Prog Lipid Res. 2012;51:314-24. Disponible en: www.pubmed.gov/22609245

\section{Revista Annual Review of Nutrition}

- Cornelis MC, Hu FB. Gene-environment interactions in the development of type 2 diabetes: recent progress and continuing challenges. Annu Rev Nutr. 2012;32:245-59. Disponible en: www.pubmed.gov/22540253

- Foltz JL, May AL, Belay B, Nihiser AJ, Dooyema CA, Blanck HM. Population-level intervention strategies and examples for obesity prevention in children. Annu Rev Nutr. 2012;32:391-415. Disponible en: www.pubmed. gov/22540254

El propósito de esta extensa revisión es proporcionar un resumen de las estrategias de intervención poblacional en relación con la prevención de la obesidad infantil y mostrar ejemplos específicos de intervención que ilustren maneras de ayudar a prevenir y controlar la obesidad en los niños mejorando la alimentación y la actividad física.

- Hall KD. Modeling metabolic adaptations and energy regulation in humans. Annu Rev Nutr. 2012;32:35-54. Disponible en: www.pubmed.gov/22540251

Esta revisión ofrece una visión general de los diversos enfoques que se han utilizado para modelar la dinámica del peso corporal y la regulación de la energía en humanos, pone de relieve varias ideas que han prestado estos modelos y propone cómo los modelos matemáticos pueden servir de guía para la investigación experimental en el futuro.

- Mayne ST, Ferrucci LM, Cartmel B. Lessons learned from randomized clinical trials of micronutrient supplementation for cancer prevention. Annu Rev Nutr. 2012;32:36990. Disponible en: www.pubmed.gov/22524186

Se analizan los resultados de los ensayos clínicos aleatorizados de los últimos 20 años sobre suplementación con micronutrientes para la prevención del cáncer. Aunque la administración de suplementos dirigidos a poblaciones con escasez del nutriente de interés podría prevenir el cáncer, esta hipótesis todavía no se ha probado formalmente. La suplementación en poblaciones con mayor estatus o que pretenda lograr exposiciones farmacológicas puede promover el cáncer. Con base en estos datos, los autores conside- 
ran que los suplementos nutritivos no se recomiendan para la prevención del cáncer en la población general.

- Parekh N, Chandran U, Bandera EV. Obesity in cancer survival. Annu Rev Nutr. 2012;32:311-42. Disponible en: www.pubmed.gov/22540252

- Shapses SA, Sukumar D. Bone metabolism in obesity and weight loss. Annu Rev Nutr. 2012;32:287-309. Disponible en: www.pubmed.gov/22809104

Pese a que tradicionalmente se ha considerado que la obesidad tiene efectos positivos en los huesos, estudios recientes señalan que compromete la calidad del hueso. Tanto la obesidad como la restricción calórica aumentan el riesgo de fractura. La disminución de peso disminuirá la masa ósea y la densidad mineral, aunque ello depende de la edad, el sexo y la adiposidad del individuo. Se puede atenuar la pérdida de hueso que la reducción de peso conlleva mediante modificaciones dietéticas, el ejercicio y ciertos fármacos.

\section{Revista The American Journal of Clinical Nutrition}

- Aune D, Chan DS, Vieira AR, Navarro Rosenblatt DA, Vieira R, Greenwood DC, et al. Dietary compared with blood concentrations of carotenoids and breast cancer risk: a systematic review and meta-analysis of prospective studies. Am J Clin Nutr. 2012;96:356-73. Disponible en: www.pubmed.gov/22760559

Debido a que los errores de medición de la ingesta de frutas y verduras en la evaluación de la dieta podrían atenuar las asociaciones con el riesgo de cáncer de mama, con lo que se explicaría la debilidad de las asociaciones observadas en los estudios epidemiológicos, los autores realizaron una revisión sistemática y metaanálisis de estudios prospectivos que relacionen tanto la ingesta dietética como las concentraciones sanguíneas de carotenoides con el riesgo de cáncer de mama. De los seis carotenoides dietéticos evaluados, sólo la ingesta de betacaroteno se asoció significativamente con menor riesgo de cáncer de mama, mientras que todos los carotenoides sanguíneos evaluados se asociaron a menor riesgo de cáncer de mama. Los autores concluyen que las concentraciones sanguíneas de carotenoides están más fuertemente asociadas con menor riesgo de cáncer de mama que los carotenoides evaluados mediante cuestionarios dietéticos. Ello indica que el uso de ciertos biomarcadores podría aclarar los resultados discordantes y débiles observados en relación con la ingesta alimentaria y el riesgo de cáncer de mama.

- Chapman CD, Benedict C, Brooks SJ, Schiöth HB. Lifestyle determinants of the drive to eat: a meta-analysis. Am J Clin Nutr. 2012;96:492-7. Disponible en: www.pubmed.gov/22836029

Ver mucho la televisión, consumir alcohol y la falta de sueño no sólo se relacionan con la obesidad, sino que contribuirían a ella fomentando una alimentación excesiva, según este metaanálisis.
- Chiuve SE, Rimm EB, Sandhu RK, Bernstein AM, Rexrode $\mathrm{KM}$, Manson JE, et al. Dietary fat quality and risk of sudden cardiac death in women. Am J Clin Nutr. 2012;96:498-507. Disponible en: www.pubmed. gov/22854398

Los autores examinan las asociaciones e interacciones entre los diferentes tipos de ácidos grasos en un estudio prospectivo de cohortes, sobre 91.981 mujeres de 34-59 años. Una mayor proporción de ácidos grasos poliinsaturados en la dieta se asoció inversamente con el riesgo de muerte súbita cardiaca, independientemente de los tradicionales factores de riesgo de cardiopatía coronaria. Estos resultados respaldan las pautas dietéticas para mejorar la calidad de la grasa dietética mediante la sustitución de la ingesta de ácidos grasos saturados por ácidos grasos poliinsaturados n-6 y n-3.

- Cogswell ME, Zhang Z, Carriquiry AL, Gunn JP, Kuklina EV, Saydah SH, et al. Sodium and potassium intakes among US adults: NHANES 2003-2008. Am J Clin Nutr. 2012;96:647-57. Disponible en: www.pubmed. gov $/ 22854410$

La gran mayoría de los adultos estadounidenses consumen demasiado sodio y muy poco potasio. Esta observación es independiente del patrón de recomendación de ingesta tomado como referencia y de las características sociodemográficas o de salud de la población.

- De Oliveira Otto MC, Mozaffarian D, Kromhout D, Bertoni AG, Sibley CT, Jacobs DR Jr, et al. Dietary intake of saturated fat by food source and incident cardiovascular disease: the Multi-Ethnic Study of Atherosclerosis. Am J Clin Nutr. 2012;96:397-404. Disponible en: www.pubmed. gov/22760560

Se siguió a voluntarios de 45-84 años $(n=5.209)$ desde 2000 a 2010 y se evaluó la incidencia de enfermedad cardiovascular (ECV). Tras ajustar por demografía, estilo de vida, dieta y factores de confusión, la ingesta de ácidos grasos saturados (AGS) a partir de productos lácteos se asoció con menor riesgo de ECV, mientras que la mayor ingesta de AGS a partir de carne se asoció con mayor riesgo de ECV. La sustitución de un $2 \%$ de la energía de los AGS de la carne por energía a partir de AGS de productos lácteos se asoció con un riesgo de ECV el $25 \%$ menor.

- Drewnowski A, Maillot M, Rehm C. Reducing the sodiumpotassium ratio in the US diet: a challenge for public health. Am J Clin Nutr. 2012;96:439-44. Disponible en: www.pubmed.gov/22760562

Menos del 0,015\% de la población americana cumple las recomendaciones de ingesta de sodio y potasio (a la vez). Los esfuerzos para mejorar esta situación son, según los autores del estudio, "extraordinarios".

- Englund-Ögge L, Brantsæter AL, Haugen M, Sengpiel V, Khatibi A, Myhre R, et al. Association between intake of artificially sweetened and sugar-sweetened beverages and preterm delivery: a large prospective cohort study. 
Am J Clin Nutr. 2012;96:552-9. Disponible en: www.pubmed.gov/22854404

En este gran estudio prospectivo, que tuvo en cuenta potenciales factores de confusión, se observa que la alta ingesta de bebidas tanto con edulcorantes artificiales como azucaradas incrementa el riesgo de parto prematuro.

- Foster GD, Shantz KL, Vander Veur SS, Oliver TL, Lent MR, Virus A, et al. A randomized trial of the effects of an almond-enriched, hypocaloric diet in the treatment of obesity. Am J Clin Nutr. 2012;96:249-54. Disponible en: www.pubmed.gov/22743313

En este ensayo aleatorizado $(n=123)$, se observa que una dieta hipocalórica sin almendras disminuye el peso corporal a los 18 meses tanto como una dieta hipocalórica con almendras. Sin embargo, el grupo asignado a consumir almendras experimentó mayores mejoras en los perfiles lipídicos.

- Gera T, Sachdev HS, Boy E. Effect of iron-fortified foods on hematologic and biological outcomes: systematic review of randomized controlled trials. Am J Clin Nutr. 2012;96:309-24. Disponible en: www.pubmed. gov/22760566

El consumo de alimentos enriquecidos con hierro genera mejoras en la hemoglobina, la ferritina y el estado de hierro de los pacientes anémicos, así como un menor riesgo de permanecer anémicos o con deficiencias de hierro, según esta revisión sistemática de ensayos aleatorizados y controlados.

- Hoffer LJ, Bistrian BR. Appropriate protein provision in critical illness: a systematic and narrative review. Am J Clin Nutr. 2012;96:591-600. Disponible en: www.pubmed. gov $/ 22811443$

Pese a que los autores reconocen que hay una necesidad urgente de estudios bien diseñados para identificar el nivel apropiado de provisión de proteínas en la enfermedad crítica y a que la mala calidad de la evidencia impide establecer conclusiones o recomendaciones clínicas, esta revisión sistemática indica que es aconsejable aportar a la mayoría de los pacientes críticos 2-2,5 g de proteína/kg de peso corporal normal/día. Actualmente, la mayoría de los adultos enfermos graves reciben menos de la mitad de la actual recomendación más habitual $(1,5 \mathrm{~g}$ de proteínas $/ \mathrm{kg} /$ día $)$ durante su estancia en una unidad de cuidados intensivos.

- Macdiarmid JI, Kyle J, Horgan GW, Loe J, Fyfe C, Johnstone A, et al. Sustainable diets for the future: Can we contribute to reducing greenhouse gas emissions by eating a healthy diet? Am J Clin Nutr. 2012;96:632-9. Disponible en: www.pubmed.gov/22854399

Los autores evaluaron si se puede lograr una reducción en las emisiones de gases de efecto invernadero a la vez que se satisfacen las necesidades dietéticas para la salud de la población. Se concluye que la dieta debe incluir lácteos y productos cárnicos en cantidades menores que en la dieta ac- tual, sin que ello afecte al coste que debe pagar el consumidor.

- Novotny JA, Gebauer SK, Baer DJ. Discrepancy between the Atwater factor predicted and empirically measured energy values of almonds in human diets. Am J Clin Nutr. 2012;96:296-301. Disponible en: www.pubmed. gov/22760558

El contenido de energía de las almendras en la dieta humana es, según este estudio, significativamente menor que el determinado por los factores de Atwater, que lo sobrestiman en un $32 \%$.

- Petry N, Egli I, Chassard C, Lacroix C, Hurrell R. Inulin modifies the bifidobacteria population, fecal lactate concentration, and fecal $\mathrm{pH}$ but does not influence iron absorption in women with low iron status. Am J Clin Nutr. 2012;96:325-31. Disponible en: www.pubmed. gov/22743314

- Romieu I, Ferrari P, Rinaldi S, Slimani N, Jenab M, Olsen $A$, et al. Dietary glycemic index and glycemic load and breast cancer risk in the European Prospective Investigation into Cancer and Nutrition (EPIC). Am J Clin Nutr. 2012;96:345-55. Disponible en: www.pubmed. gov $/ 22760570$

El índice glucémico, la carga glucémica (CG) o la ingesta de hidratos de carbono no se asocian con el riesgo de cáncer de mama, aunque se observa una asociación positiva entre una dieta con alta CG y el riesgo de cáncer de mama en mujeres posmenopáusicas.

- Shay CM, Van Horn L, Stamler J, Dyer AR, Brown IJ, Chan $Q$, et al. Food and nutrient intakes and their associations with lower BMI in middle-aged US adults: the International Study of Macro-/Micronutrients and Blood Pressure (INTERMAP). Am J Clin Nutr. 2012;96:483-91. Disponible en: www.pubmed.gov/22854407

Una mayor ingesta de alimentos con hidratos de carbono y alta densidad nutricional, pero pobres en proteína animal y ácidos grasos saturados, se asocia a una menor ingesta energética, una ingesta de micronutrientes más favorable y un menor índice de masa corporal según este gran estudio transversal.

- Sinha R, Cross AJ, Daniel CR, Graubard BI, Wu JW, Hollenbeck AR, et al. Caffeinated and decaffeinated coffee and tea intakes and risk of colorectal cancer in a large prospective study. Am J Clin Nutr. 2012;96:374-81. Disponible en: www.pubmed.gov/22695871

- Sylvetsky AC, Welsh JA, Brown RJ, Vos MB. Low-calorie sweetener consumption is increasing in the United States. Am J Clin Nutr. 2012;96:640-6. Disponible en: www. pubmed.gov/22854409

- Tobias DK, Zhang C, Chavarro J, Bowers K, Rich-Edwards $\mathrm{J}$, Rosner B, et al. Prepregnancy adherence to dietary patterns and lower risk of gestational diabetes mellitus. Am J Clin Nutr. 2012;96:289-95. Disponible en: www.pubmed.gov/22760563 
- Verstraeten R, Roberfroid D, Lachat C, Leroy JL, Holdsworth M, Maes L, et al. Effectiveness of preventive school-based obesity interventions in low- and middleincome countries: a systematic review. Am J Clin Nutr. 2012;96:415-38. Disponible en: www.pubmed. gov/22760565

- Vlaardingerbroek H, Veldhorst MA, Spronk S, Van den Akker CH, Van Goudoever JB. Parenteral lipid administration to very-low-birth-weight infants-early introduction of lipids and use of new lipid emulsions: a systematic review and meta-analysis. Am J Clin Nutr. 2012;96:25568. Disponible en: www.pubmed.gov/22743312

- Wark PA, Lau R, Norat T, Kampman E. Magnesium intake and colorectal tumor risk: a case-control study and metaanalysis. Am J Clin Nutr. 2012;96:622-31. Disponible en: www.pubmed.gov/22854408

Este estudio de casos y controles, combinado con un metaanálisis de la literatura, apoya la hipótesis de que una mayor ingesta de alimentos ricos en magnesio se asocia con menor riesgo de cánceres colorrectales.

\section{Revista Nutrition Research Reviews}

No se han publicado artículos en las fechas evaluadas en la presente revisión.

\section{Revista Journal of the American Dietetic Association}

- Britten P, Cleveland LE, Koegel KL, Kuczynski KJ, NickolsRichardson SM. Updated US Department of Agriculture Food Patterns Meet Goals of the 2010 Dietary Guidelines. J Acad Nutr Diet. 2012;112:1648-55. Disponible en: www. pubmed.gov/22853987

- Bruening M, Eisenberg M, Maclehose R, Nanney MS, Story $M$, Neumark-Sztainer D. Relationship between adolescents' and their friends' eating behaviors: breakfast, fruit, vegetable, whole-grain, and dairy intake. J Acad Nutr Diet. 2012;112:1608-13. Disponible en: www.pubmed.gov/23017570

- De Lauzon-Guillain B, Oliveira A, Charles MA, Grammatikaki E, Jones L, Rigal N, et al. A review of methods to assess parental feeding practices and preschool children's eating behavior: the need for further development of tools. J Acad Nutr Diet. 2012;112:1578-602.e8. Disponible en: www.pubmed.gov/23017568

- Gaesser GA, Angadi SS. Gluten-free diet: imprudent dietary advice for the general population? J Acad Nutr Diet. 2012;112:1330-3. Disponible en: www.pubmed. gov/22939437

No hay evidencia, según los autores, de que seguir una dieta sin gluten proporcione alguna clase de beneficio significativo en la población general. Se señala, asimismo, que sí hay evidencia de que dicha modificación dietética, a no ser que esté justificada médicamente, puede afectar negativamente a la salud intestinal de los pacientes sin enfermedad celiaca o sin sensibilidad al gluten.
- Kong A, Beresford SA, Alfano CM, Foster-Schubert KE, Neuhouser ML, Johnson DB, et al. Self-monitoring and eating-related behaviors are associated with 12-month weight loss in postmenopausal overweight-to-obese women. J Acad Nutr Diet. 2012;112:1428-35. Disponible en: www.pubmed.gov/22795495

El autocontrol (autopesaje - pesarse a menudo-, seguir un diario dietético y ciertos aspectos relacionados con la alimentación como preparar comidas en casa) podría mejorar la pérdida de peso de las mujeres posmenopáusicas, según este estudio llevado a cabo durante 12 meses con 123 voluntarias.

- Leong SL, Madden C, Gray A, Horwath C. Self-determined, autonomous regulation of eating behavior is related to lower body mass index in a nationwide survey of middle-aged women. J Acad Nutr Diet. 2012;112:1337-46. Disponible en: www.pubmed.gov/22939438

- Levine J, Wolf RL, Chinn C, Edelstein BL. MySmileBuddy: An iPad-based interactive program to assess dietary risk for early childhood caries. J Acad Nutr Diet. 2012;112:1539-42. Disponible en: www.pubmed.gov/ 23017564

- Mehta UJ, Siega-Riz AM, Herring AH, Adair LS, Bentley $M E$. Pregravid body mass index is associated with early introduction of complementary foods. J Acad Nutr Diet. 2012;112:1374-9. Disponible en: www.pubmed.gov/ 22939440

Las mujeres con sobrepeso u obesidad serían más proclives a incorporar la alimentación complementaria a sus bebés de manera prematura, según este estudio de cohorte prospectivo (2001 hasta 2005) que reclutó a mujeres embarazadas de entre 15 y 20 semanas de gestación, con un seguimiento a 12 meses después del parto $(n=550)$.

- Oza-Frank R, Zavodny M, Cunningham SA. Beverage displacement between elementary and middle school, 20042007. J Acad Nutr Diet. 2012;112:1390-6. Disponible en: www.pubmed.gov/22818245; PubMed Central PMCID: PMC3432667.

- Palmer CA, Gilbert JA. Position of the Academy of Nutrition and Dietetics: the impact of fluoride on health. J Acad Nutr Diet. 2012;112:1443-53. Disponible en: www. pubmed.gov/22939444

Se trata de la postura de la Academia de Nutrición y Dietética, que apoya la utilización óptima de flúor sistémico y tópico como una medida de salud pública importante para promover la salud oral y general durante toda la vida. El flúor es un elemento importante en la mineralización de los huesos y los dientes. Su uso adecuado ha reducido la caries dental y la discapacidad asociada. La caries dental sigue siendo la enfermedad crónica más frecuente en niños y afecta a todos los grupos de edad de la población. Los Centros para el Control y Prevención de Enfermedades han citado la fluoración del agua como una de las diez medidas de salud pública más importantes del siglo XXI. Actualmente más del $72 \%$ de la población de Estados Unidos se beneficia de la fluoración del agua. Por el momento, el uso de altas 
dosis de flúor para la prevención de la osteoporosis se considera experimental.

- Pan L, Sherry B, Njai R, Blanck HM. Food insecurity is associated with obesity among US adults in 12 states. J Acad Nutr Diet. 2012;112:1403-9. Disponible en: www. pubmed.gov/22939441

Los adultos que padecen inseguridad alimentaria (población general y la mayoría de los subgrupos de población) tienen un $32 \%$ más probabilidades de padecer obesidad según este estudio, que contó con una muestra de 66.553 adultos estadounidenses (de doce estados).

- Park S, Blanck HM, Sherry B, Brener N, O'Toole T. Factors associated with low water intake among US high school students - National Youth Physical Activity and Nutrition Study, 2010. J Acad Nutr Diet. 2012;112:1421-7. Disponible en: www.pubmed.gov/22749261

- Raynor HA, Looney SM, Steeves EA, Spence M, Gorin AA. The effects of an energy density prescription on diet quality and weight loss: a pilot randomized controlled trial. J Acad Nutr Diet. 2012;112:1397-402. Disponible en: www.pubmed.gov/22575072

- Thompson T, Grace T. Gluten in cosmetics: is there a reason for concern? J Acad Nutr Diet. 2012;112:1316-23. Disponible en: www.pubmed.gov/22939435
- Turner L, Chriqui JF, Chaloupka FJ. Food as a reward in the classroom: school district policies are associated with practices in US public elementary schools. J Acad Nutr Diet. 2012;112:1436-42. Disponible en: www.pubmed. gov/22640775

El uso de los alimentos como recompensa por el buen comportamiento como estudiante o el buen rendimiento académico está desaconsejado por muchas organizaciones. Pese a ello, esta práctica sigue ocurriendo en las escuelas. Ese estudio evaluó el uso de la comida como premio en las escuelas primarias de Estados Unidos, y observó esta práctica sólo está prohibida en el $11,9 \%$ de los distritos evaluados. Según los autores, se debe fortalecer las políticas al respecto.

- Wang Y, Yang M, Lee SG, Davis CG, Koo SI, Chun OK. Dietary total antioxidant capacity is associated with diet and plasma antioxidant status in healthy young adults. $\mathrm{J}$ Acad Nutr Diet. 2012;112:1626-35. Disponible en: www. pubmed.gov/23017573

- Yaroch AL, Tooze J, Thompson FE, Blanck HM, Thompson OM, Colón-Ramos U, et al. Evaluation of three short dietary instruments to assess fruit and vegetable intake: the National Cancer Institute's food attitudes and behaviors survey. J Acad Nutr Diet. 2012;112:1570-7. Disponible en: www.pubmed.gov/23017567 